\title{
Luces y sombras de la educación infantil 0-3 en Andalucía: condiciones de acceso y de escolarización ${ }^{1}$
}

\author{
Lights and Shadows of Early Childhood Education (0 to 3) in Andalusia: \\ Acces Conditions to Reception and Schooling
} Manuel Ángel Río Ruiz, Rubén Martín Gimeno, María Ortega Gómez²

\section{Resumen}

El artículo examina diferentes facetas de la Educación Infantil 0-3 en Andalucía. Se analizan las características e historia reciente del modelo implantado aquí en el contexto de la Educación 0-3 estatal. El análisis revela una gran expansión reciente de la escolaridad en todos los años de este ciclo, pero basada en la multiplicación de una oferta de centros infantiles privados concertados infrafinanciados, en dificultades económicas, muy condicionados a cumplir mínimos de proyectos asistenciales. Todo ello limita muchas veces la calidad y eficacia pedagógica que se viene crecientemente demandando a este ciclo. El análisis también identifica exclusiones por motivos económicos, pero que afectan menos a las familias más vulnerables de menos recursos por debajo del umbral de la pobreza que a las familias de clases populares que se autoexcluyen o retrasan al máximo la matriculación al carecer de bonificaciones, incentivos económicos y derechos a plazas gratuitas.

\section{Palabras clave}

Educación Infantil 0-3, privatización educativa, calidad educativa, desigualdad educativa.

\section{Abstract}

This article examines different aspects of 0-3-year-old pre-school education in Andalusia. It analyses the characteristics and recent history of the model implemented here in the context of state-wide $0-3$-year-old education. The analysis reveals a great recent expansion of schooling in all the years of this cycle but based on the proliferation of an offer of underfinanced private subsidized children's centres, in economic difficulties, very conditioned by this to meet minimum assistance projects. All of this often limits the pedagogical quality and efficiency that are increasingly demanded for this cycle. The analysis also identifies exclusions for economic reasons, but these have a lower impact on the most vulnerable families with fewer resources below the poverty line than families from the lower classes who exclude themselves or delay enrolment as much as possible due to the lack of subsidies, economic incentives, and the right to free places.

\section{Keywords}

0-3-year-old pre-school education, educational privatization, educational quality, educational inequality.

\section{Cómo citar/Citation}

Río Ruiz, Manuel Ángel; Martín Gimeno, Rubén y Ortega Gómez, María (2022). Luces y sombras de la educación infantil 0-3 en Andalucía: condiciones de acceso y de escolarización. Revista de Sociología de la Educación-RASE, 15 (1), 127-151. http://dx.doi.org/10.7203/ RASE.15.1.22990.

\footnotetext{
Este texto integra parte de los resultados y datos actualizados de la investigación «Estudio sobre acceso equitativo y calidad en Educación Infantil de primer ciclo (0-3 años) en Andalucía», financiado en 2019 por Save the Children - Andalucía, dirigido por el primer autor y en el que han participado los otros dos. Agradecemos a Antonio Echaves García su colaboración en la explotación de algunos de los datos que utilizamos.

2 Manuel Ángel Río Ruiz, Universidad de Sevilla, manurio@us.es; Rubén Martín Gimeno, Universidad Pablo Olavide de Sevilla, rmargim@upo.es y María Ortega Gómez, Universidad de Sevilla, mariaortega.uni@gmail.com.
} 


\section{Introducción}

Dos circunstancias han conformado un relativo consenso, incluso entre partidos políticos, en torno a la Educación Infantil de 0 a 3 años en España (a partir de ahora EI-03). En primer lugar, la acumulación de evidencias sobre el impacto pedagógico de la EI-03 (Van Belle, 2016; Mancebón et al., 2018) convertida en nueva herramienta de salvación social mediante lo escolar que iría mucho más allá de estrategias económicas familiares de conciliación y custodia: sería una etapa de educabilidad con entidad propia clave para anticiparnos a los costes del fracaso escolar (Heckman, 2011; Van Huizen et al., 2016) y hacer frente a los efectos de la pobreza infantil que crece en España (León y Muñoz, 2016; González et al., 2019). En segundo lugar, una expectativa y demanda creciente de estos servicios desde hace más de una década, materializada en un aumento progresivo — en algunas comunidades acelerado- de las tasas netas de matriculación. Al menos hasta que llegó la Covid-19.

Literalmente «la extensión de la gratuidad en la etapa Infantil de 0 a 3 años» figura en el programa de la derecha y de la izquierda. Más allá de lo programado, ubicados en el más gris terreno de los hechos, los dos grandes partidos que se han repartido la gestión de la educación reúnen un modelo bastante homologable: contradicen ambos sus programas. Coinciden, pero en sus políticas autonómicas de expansión del sector privado en detrimento de la potenciación de la red pública garantizadora de la gratuidad. La notable multiplicación de la demanda y de la matriculación en EI-03 se ha gestionado por la mayoría de gobiernos autonómicos de tal manera que, actualmente, la mayor parte de los centros de EI-03 españoles son privados y buena parte de las familias se autoexcluyen del ciclo por motivos económicos, aunque también por desequilibrios de oferta y desajustes entre permisos parentales, jornadas laborales y escuelas infantiles (Save the Children, 2019; Romero-Balsas, Rogero-García y Meil, 2022).

Con la mayor población entre 0 y 3 años, Andalucía, la región cuya EI-03 examina este artículo, representa un caso de creación, consolidación y naturalización ideológica de un modelo privatizado basado en bonificaciones a parte de las familias de menos ingresos, así como en la regulación de los precios que cobran las escuelas infantiles adheridas a conciertos con la Consejería de Educación. Esta política deja fuera por motivos económicos a buena parte de la población precaria estabilizada de Andalucía. El modelo que se mantiene actualmente en esta tierra es exactamente el heredado de anteriores gobiernos, de distinto signo político, que hoy desde la oposición denuncian el que fue también su «sistema privatizador» creado para responder a la demanda creciente de EI-03. Demanda que, pese a lo dicho arriba, se materializa hoy en una de las tasas netas de matriculación en EI-03 de las más altas del Estado, siendo esta última una de las mejores noticias que pueden darse sobre enseñanzas preuniversitarias en Andalucía.

El objetivo de este artículo es examinar las sombras y luces arriba apenas esbozadas. Para ello, el primer apartado tras la metodología ofrece algunos datos recientes y balances de la situación de la EI-03 en el conjunto de España. El fin: situar a quienes lean en un marco más amplio que el andaluz bajo la advertencia, no obstante, de que lo que más unifica la EI-03 española es el dominio de la provisión privada ${ }^{3}$, pero dentro de la disparidad entre comunidades en tipos y cuantías de ayudas y bonificaciones familiares, en gasto público y privado en el ciclo, así como en tasas de escolarización a distintas edades.

En segundo lugar, se analizan las condiciones de acceso a la EI-03 andaluza. Por un lado, la evolución de la escolarización y de la provisión de plazas, atendiendo a las características de la oferta que la absorbe. Por otro, se identifican algunos factores que limitan el acceso a la EI-03 en Andalucía.

3 A excepción del País Vasco y en menor medida Cataluña y Aragón. 
En tercer lugar, se analizan las condiciones de escolarización en la EI-03 andaluza. Se atiende a diferentes dimensiones que permiten dar cuenta y razones de algunos déficits detectados en la calidad del ciclo. Déficits asociados la mayoría a la infrafinanciación del sistema.

En el momento de escribir este artículo dos cuestiones quedan para futuros análisis. No se han podido explicar, aunque sí identificar, los efectos de la Covid-19 sobre la EI-03 andaluza ${ }^{4}$. También desborda el objetivo y espacio de este trabajo analizar las oportunidades y desafíos a los que se expone el dominante mercado privado de EI-03 de Andalucía. Sistema que, si no se reformula y se refinancia de manera ambiciosa, se encamina a una gran crisis por tres razones principales. La primera: los efectos de la caída de la fecundidad y de la sobreoferta de plazas, algo que incluso afecta ya a algunas escuelas infantiles públicas. La segunda: la insuficiencia de las cuantías recibidas por los centros por familias que ocupan plazas bonificadas. La tercera: la gran competencia que para este mercado educativo privatizado supondrá la obligación europea de destinar parte de los nuevos fondos europeos exclusivamente a la creación de miles de plazas públicas gratuitas ${ }^{5}$. Andalucía reúne, gracias a los nuevos fondos europeos, una gran oportunidad para multiplicar su red de plazas públicas, así como para reinvertir en la existente y revertir privatizaciones de escuelas infantiles municipales, por ejemplo. Dicha oportunidad conlleva, en cambio, el dilema y el desafío de cómo desmontar o mantener a flote un sistema privatizado de 15000 empleos feminizados ${ }^{6}$. Las decisiones que se tomen para el futuro de la EI-03 andaluza debieran estar informadas sociológicamente. Este trabajo pretende contribuir a ello.

\section{Metodología}

El trabajo se sustenta en la integración de entrevistas a profesionales de la EI-03 andaluza con la explotación de datos procedentes de estadísticas públicas educativas, del registro estadístico de la administración educativa andaluza que cedió algunos datos, así como de encuestas e informes estatales e internacionales de organismos públicos y entidades sociales.

El análisis de la EI-03 se ve dificultado por la dispersión de los datos entre las autonomías responsables. Son pocas e incompletas las fuentes oficiales que reportan datos sobre esta etapa. Incluso la frecuentemente utilizada para análisis estatales Encuesta de Condiciones de Vida (ECV) presenta limitaciones para analizar la estructuración social de la EI-03. La principal: no reúne la muestra estadística necesaria para explotar con validez los datos desagregados por comunidades autónomas. De forma similar, las estadísticas ministeriales sobre nivel de ayudas (no son becas) y gasto público en educación infantil no permiten diferenciar qué se destina a primer y segundo ciclo, lo que empobrece los análisis.

Ante las lagunas en algunos de los datos disponibles, la reconstrucción de experiencias profesionales a través de 15 entrevistas ( 2 en grupo) representa, para nuestro análisis y en nuestras conclusiones, mucho más que el recurso a datos cualitativos como función retórica de las ilustraciones cuantitativas, o como muleta que proporciona apoyo vívido a los datos estadísticos. Principalmente se ha entrevistado a directoras/es de centros infantiles, pero también a inspectores/as, representantes sindicales y de la patronal, así como a técnicos/as de administraciones con responsabilidades de gestión en EI-03. El análisis se orientó

4 En España el número de matriculaciones se ha reducido un $17 \%$ en el curso 2020/21, lo que ha llevado al cierre de centros e inmediatos despidos de profesionales, casi un $99 \%$ de mujeres (Save The Children, 2021).

5 Se estima a nivel estatal unas 64.000 nuevas plazas públicas financiadas por Fondos Europeos. El peso poblacional de Andalucía la convertirá en una de las principales beneficiarias en esta materia también.

6 Como ya ha sucedido en el mercado de la Formación Profesional en Andalucía, no son descartables los riesgos de penetración de fondos de inversión en este sector, donde ya operan empresas de servicios como CLECE que han venido obteniendo concesiones de escuelas públicas municipales. 
por procedimientos y principios de análisis sociológico del discurso como la saturación cualitativa a través de la comparación continuada del corpus de entrevistas que se iba reuniendo. Se utilizaron plantillas de análisis estructuradas en función de los tópicos que arrojaba la comparación entre entrevistas, así como de los objetivos específicos de investigación que se habían integrado en diferentes puntos del guion de las entrevistas. Las entrevistas permitieron también integrar información sobre barreras de acceso y condiciones de escolarización a las que, algunas veces, apuntaban los otros datos que se recopilaban de distintas fuentes administrativas y estadísticas. Las personas entrevistadas en distintas capitales andaluzas aparecen con sus respectivos códigos en la Tabla I:

Tabla I. Informantes entrevistados/as y cargos en El-03

\begin{tabular}{|c|c|c|}
\hline & CÓDI-IDENTI. INFORMANTES & CARGOS \\
\hline 1 & DIREI.Privada.Sev & Directora Centro Adherido \\
\hline 2 & DIRE2.Pública.Sev & Director Escuela Infantil Pública \\
\hline 3 & DIRE3.Pública-Munic.Mál & Directora Escuela Infantil Pública Municipal \\
\hline 4 & DIRE4.Pública-Junta.Sev & Directora Escuela Infantil Pública \\
\hline 5 & DIRE5.Privada.Hue & Directora Centro Adherido \\
\hline 6 & DIRE6.Privada.Mál & Directora Centro Adherido \\
\hline 7 & DIRE7.Pública-Munic.Gran & Directora Red Escuelas Municipales. \\
\hline \multirow[t]{3}{*}{8} & REPREPATRONAL.Sev & Presidenta de Patronal El-03, directora centro El-03 \\
\hline & & Vicepresidenta de Patronal El-03, directora centro El-03 \\
\hline & & Secretaria y Tesorera de Patronal El-03, directora centro El-03 \\
\hline 9 & TÉCNII.Ayto.Mál & Técnico Menores Escuelas Infantiles Municipales \\
\hline \multirow[t]{2}{*}{10} & TÉCNII.Junta.Sev & Jefe de Servicios de Planificación Educativa de Infantil. \\
\hline & TÉCNI2.Junta.Sev & Técnica de Planificación Educativa de Infantil \\
\hline 11 & INSPEDUI.Sev & Inspector Educación \\
\hline 12 & INSPEDU2.Sev & Inspectora Educación \\
\hline 13 & REPRESINDICA.Sev & Representante Sindical El-03, profesora El \\
\hline 14 & EXPERUNIV.Sev & Catedrático Educación, experto en El \\
\hline 15 & REPREFEDEAMPAS.Córd & Presidenta Fede. Asoc. Madres Padres \\
\hline
\end{tabular}

\section{La Educación Infantil 0-3 en España: algunos apuntes y datos}

En España, la tasa de escolarización en el primer ciclo de EI-03 no ha dejado de crecer en la última década, alcanzando el 39,6\% y situándose por encima de la media de la OCDE $(24,9 \%)$ y de la Unión Europea (22,4\%) (OCDE, 2021). No obstante, la posibilidad de avance es aún notable, sobre todo entre 0-1 años. En el último curso, el número total de matriculados en el segundo ciclo de infantil fue de más de 1000000 frente a 390425 matriculados en el primer ciclo (MEFP, 2021: 18).

Como se sabe, desde hace más de una década la tasa neta de escolarización a los tres años alcanza a casi la totalidad de la población de esa edad. En el curso 2015-2016, a los 2 años, más de la mitad de menores se encontraba escolarizado. La tendencia al alza se observa también en la tasa correspondiente a las edades inferiores a 2 años, que en 2015-2016 alcanzaba el 46,8\%. (MEFP, 2021:18). Como muestra la Tabla II, las tasas de escolarización neta en EI-03 han seguido aumentando. 
Tabla II. Evolución de las tasas de escolaridad en Educación Infantil (menos de 1 año a 3 años). Año 2020-20217

\begin{tabular}{lccc}
\hline & $2010-2011$ & $2015-2016$ & $2020-2021$ \\
\hline Menos de 1 año & 8,8 & 10,1 & 11,0 \\
\hline 1 año & 29,9 & 36,7 & 37,8 \\
\hline 2 años & 46,3 & 57,2 & 56,4 \\
\hline 3 años & 95,6 & 96,2 & 94,1 \\
\hline Total primer ciclo & 432222 & 445262 & 390425 \\
\hline
\end{tabular}

Fuente: elaboración propia a partir de MEFP (2021): 18.

Aunque las tendencias sociales, más que las políticas públicas, han producido una expansión notoria de esta etapa, subsisten déficits compartidos por prácticamente todos los sistemas autonómicos y las diferencias en precios y regulación de estos servicios son notables (Ibáñez y León, 2014; Martín-Sonseca et al, 2016; Colom y Gabaldón, 2016; Jiménez Delgado et al., 2016).

A pesar de las demandas iniciales de las familias (CIS, 2009), la expansión del ciclo se ha producido, en la mayoría de comunidades autónomas, a través de conciertos con centros de titularidad privada (Save the Children, 2016: 62). La multiplicación de la demanda se ha respondido incrementando los desequilibrios entre oferta pública y privada a favor de la segunda. Por otro lado, el acceso desigual en función del nivel de ingresos que presenta el sistema de Educación Infantil en España ha tratado de solventarse a través de sistemas de bonificaciones y de subvenciones públicas autonómicas. Los resultados son magros. Como recoge la Tabla III, apenas el 16\% del alumnado de EI recibía «becas y ayudas» (Valdés et al., 2021: 76). Además, el gasto en ayudas a la escolarización 0-3 presenta grandes contrastes entre comunidades (Valdés et al., 2021: 77).

Tabla III. Becas y ayudas concedidas en E. Infantil por Comunidades Autónomas. 2018-2019

\begin{tabular}{lcccc}
\hline $\begin{array}{c}\text { \% BECARIOS } \\
\text { RESPECTO AL } \\
\text { ALUMNADO } \\
\text { MATRICULADO }\end{array}$ & $\begin{array}{c}\text { IMPORTE } \\
\text { POR BECARIOS } \\
\text { (EUROS) }\end{array}$ & $\begin{array}{c}\text { IMPORTE TOTAL DE } \\
\text { BECAS (MILLONES } \\
\text { DE EUROS) }\end{array}$ & $\begin{array}{c}\text { ALUMN. } \\
\text { MATRICULADO }\end{array}$ & $\begin{array}{c}\text { ALUMNADO } \\
\text { BECARIO }\end{array}$ \\
\hline España & 16,4 & 581,3 & 166,5 & 286375 \\
\hline Andalucía & 20,1 & 521 & & \\
\hline Baleares & 1,1 & 1.176 & & \\
\hline Canarias & 42,2 & 262 & & \\
\hline Castilla-La Mancha & 7,8 & 422 & & \\
\hline Cataluña & 14,0 & 566 & & \\
\hline Comunidad Valenciana & 20,5 & 497 & & \\
\hline Extremadura & 1,5 & 732 & \\
\hline Madrid & 21,5 & 786 & \\
\hline País Vasco & 20,9 & 626 & \\
\hline
\end{tabular}

Fuente: elaboración propia a partir de datos de Valdés et al. (2021): 76-77. 
La siguiente tabla ofrece un resumen del estado de conocimientos sobre la EI-03 en España:

Tabla IV. Resumen de la El-03 en España: fortalezas y debilidades

\begin{tabular}{|c|c|c|}
\hline DIMENSIÓN & FORTALEZAS & DEBILIDADES \\
\hline \multirow[t]{3}{*}{ Niveles de escolarización } & \multirow{2}{*}{$\begin{array}{l}\text { Cumplimos Pacto de Barcelona (2002): por encima de la OCDE y de la Unión } \\
\text { Europea en escolarización 0-3 }\end{array}$} & Escasa escolarización entre 0 y 2 años frente a $2-3$ años \\
\hline & & Notables diferencias entre CC. A. en tasas de matriculación \\
\hline & Expansión: duplicación de la tasa en aprox. una década & Aún lejos de la universalización lograda en poco tiempo entre 3 y 6 años \\
\hline \multirow[t]{4}{*}{ (Des)Ajuste entre oferta y demanda } & $\begin{array}{l}\text { Aumento de la demanda parental de servicios correspondido con mayor oferta de } \\
\text { plazas }\end{array}$ & $\begin{array}{l}\text { Debilidad de la red pública, con diferencias entre CC. AA.: más públicas en Cataluña } \\
\text { y Euskadi }\end{array}$ \\
\hline & \multirow[t]{3}{*}{ Logros en disponibilidad y ocupación de plazas } & $\begin{array}{l}\text { Expansión de los conciertos con centros privados experimentadores de crecientes } \\
\text { problemas de financiación }\end{array}$ \\
\hline & & $\begin{array}{l}\text { Escasez de plazas públicas frente a la (cada vez más en más lugares) sobreoferta } \\
\text { privada }\end{array}$ \\
\hline & & Desajustes entre permisos parentales, horarios y escuelas infantiles \\
\hline \multirow[t]{5}{*}{ Inversión } & \multirow[t]{5}{*}{ Sistemas de bonificaciones y subvenciones públicas } & Escasa cobertura y gasto en ayudas a la escolarización con contrastes entre CC. AA. \\
\hline & & De los mayores gastos privados de la UE en El-03 \\
\hline & & Menor inversión en red pública: reúne costes mayores para СC. AA. responsables \\
\hline & & $\begin{array}{l}\text { Infrafinanciación e irregularidad de Planes oficiales: Educa } 3 \text { (2008-2010) } 100 \\
\text { millones y sin continuidad }\end{array}$ \\
\hline & & $\begin{array}{l}\text { Riesgos de traspaso en red de escuelas infantiles privadas a fondos de inversión: } \\
\text { riesgos de mayor mercantilización educativa }\end{array}$ \\
\hline \multirow[t]{4}{*}{ Accesibilidad y equidad } & \multirow[t]{4}{*}{ Proyectos legislativos de universalización de la etapa } & $\begin{array}{l}\text { Persistencia de desequilibrios territoriales en la implantación de centros: barrios } \\
\text { vulnerables no atraen oferta privada }\end{array}$ \\
\hline & & Variaciones notables de precios de los por CC. АA. \\
\hline & & $\begin{array}{l}\text { Más del } 50 \% \text { de las familias pagan íntegramente los servicios de E1-03. Menos } \\
\text { del } 25 \% \text { ocupa plazas gratuitas }\end{array}$ \\
\hline & & $\begin{array}{l}\text { Desigualdades de presencia en función de ingresos y nivel de estudios: a mayor } \\
\text { renta y mayor nivel educativo más probabilidades de escolarizar en } 03\end{array}$ \\
\hline \multirow[t]{2}{*}{ Calidad } & $\begin{array}{l}\text { Algunas medidas LOMLOE refuerzan la dimensión pedagógica: coordinación entre } \\
\text { ciclos, desarrollo y diferenciación competencial }\end{array}$ & \multirow{2}{*}{$\begin{array}{l}\text { Cumplimiento del proyecto asistencial en detrimento de la eficacia y calidad } \\
\text { pedagógica del ciclo: consecuencia de la infrafinanciación y del modelo de negocio } \\
\text { que fomenta precariedad estructural del sistema (salarios bajos en la privada, } \\
\text { dificultades para formación continua...) }\end{array}$} \\
\hline & $\begin{array}{l}\text { Sumisión a la razón pedagógica: crece en los poderes públicos la ilegitimidad de } \\
\text { visiones «no es educación, es conciliación» }\end{array}$ & \\
\hline
\end{tabular}

Fuente: elaboración propia.

\section{La Educación Infantil 0-3 en Andalucía}

\subsection{Expansión, pero bajo una apuesta privatizadora}

Pese a registrar aún durante los noventa del pasado siglo tasas de escolarización sensiblemente inferiores a las del conjunto del Estado, Andalucía lleva experimentando desde hace tres lustros un incremento acelerado de la tasa neta de escolarización en EI-03. Pese a sus niveles de pobreza y de paro, sobre todo femenino, la región figura por encima de la media estatal en tasa neta de escolaridad entre 0 y 2 años, con un 48\% de matriculación justo antes de la Pandemia. El gráfico 1 representa uno de los datos más positivos de la educación andaluza, tan acostumbrada a malas noticias. No obstante, Andalucía también registra a causa de la Covid-19 un descenso acusado de la matriculación en EI-03 similar al resto de España. 


\section{Gráfico 1. Evolución Tasa neta de escolaridad 0-2 años. Andalucía y España. 1991- 2021*}

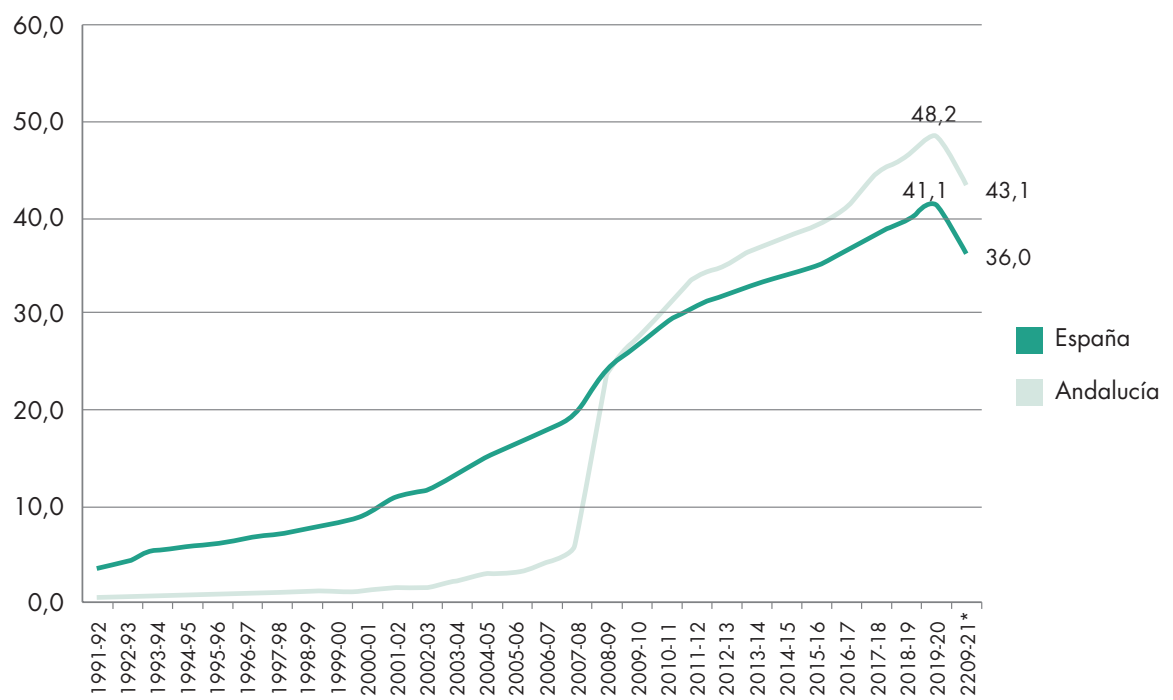

Fuente: Ministerio de Educación y Formación Profesional. Las cifras de la educación en España.

*Los datos del curso 2020-2021 son datos avance

La escolarización en EI-03 de Andalucía se sitúa por encima de la media estatal en todos los años del ciclo. Y las diferencias se acentúan a medida que avanza éste. Por tanto, puede hablarse de una gran expansión de la EI-03 andaluza, como representaba el señalado Gráfico 1. En él puede observarse también un despegue a partir del curso 2007-2008, el cual se acelera y consolida desde el curso 20092010. Influye decisivamente en todo ello la aprobación del Decreto de 2008 que regula y ordena la EI-03 en Andalucía ${ }^{8}$. Las competencias pasan de Bienestar Social a Educación, quien además absorbe buena parte de las competencias que reunían los Ayuntamientos. Además de fijar las condiciones de escolarización a cumplir por los centros, tanto públicos como privados, dicha normativa sienta las bases del incremento graficado al convertir la dotación de plazas en este ciclo en un derecho para las familias?.

Los cambios más importantes en los últimos años se han producido entre 0 y 1 año. Si tomamos como referencia el curso 2016-2017, se observaba que la tasa de escolarización andaluza en las edades de 1 y 2 años era significativamente mayor que las medias de España. En cambio, en dicho curso Andalucía se situaba por debajo en la tasa de 0-1 año (10,9 en España y 9,8 en Andalucía). Esto también ha cambiado. Como se observa en el gráfico 2a, para el curso 2020-2021 la tendencia al alza en las matriculaciones en la edad más tempran ${ }^{10}$ ha sido más intensa en Andalucía.

8 Decreto 428/2008, de 29 de julio, por el que se establece la ordenación y las enseñanzas correspondientes a la Educación Infantil en Andalucía. (BOJA 19-8-2008). https://www.juntadeandalucia.es.

9 Además del citado Decreto autonómico sobre el despegue de la EI-03 andaluza influirá, sobre todo en la creación de centros públicos, el Plan Educa 3 bajo la Presidencia de Zapatero. Abortado además por la crisis en 2010, este plan tendrá escasos efectos económicos: apenas repartió los dos años anteriores 100 millones de euros entre todas las administraciones para dotar plazas públicas figurando el criterio poblacional como el principal de reparto. Por su parte, la Ley Orgánica de Educación (LOE), de 2006, instaba a las administraciones a desarrollar una oferta suficiente de plazas en EI-03 a través de convenios con las corporaciones locales, otras administraciones o entidades privadas sin fines de lucro. Frente a la declaración de intenciones, en la práctica la mayor parte de administraciones apostaron por la privatización y naturalización como negocio privado de los socialmente demandados servicios de EI-03.

10 La escolarización es posible en Andalucía a partir de las 16 semanas. 


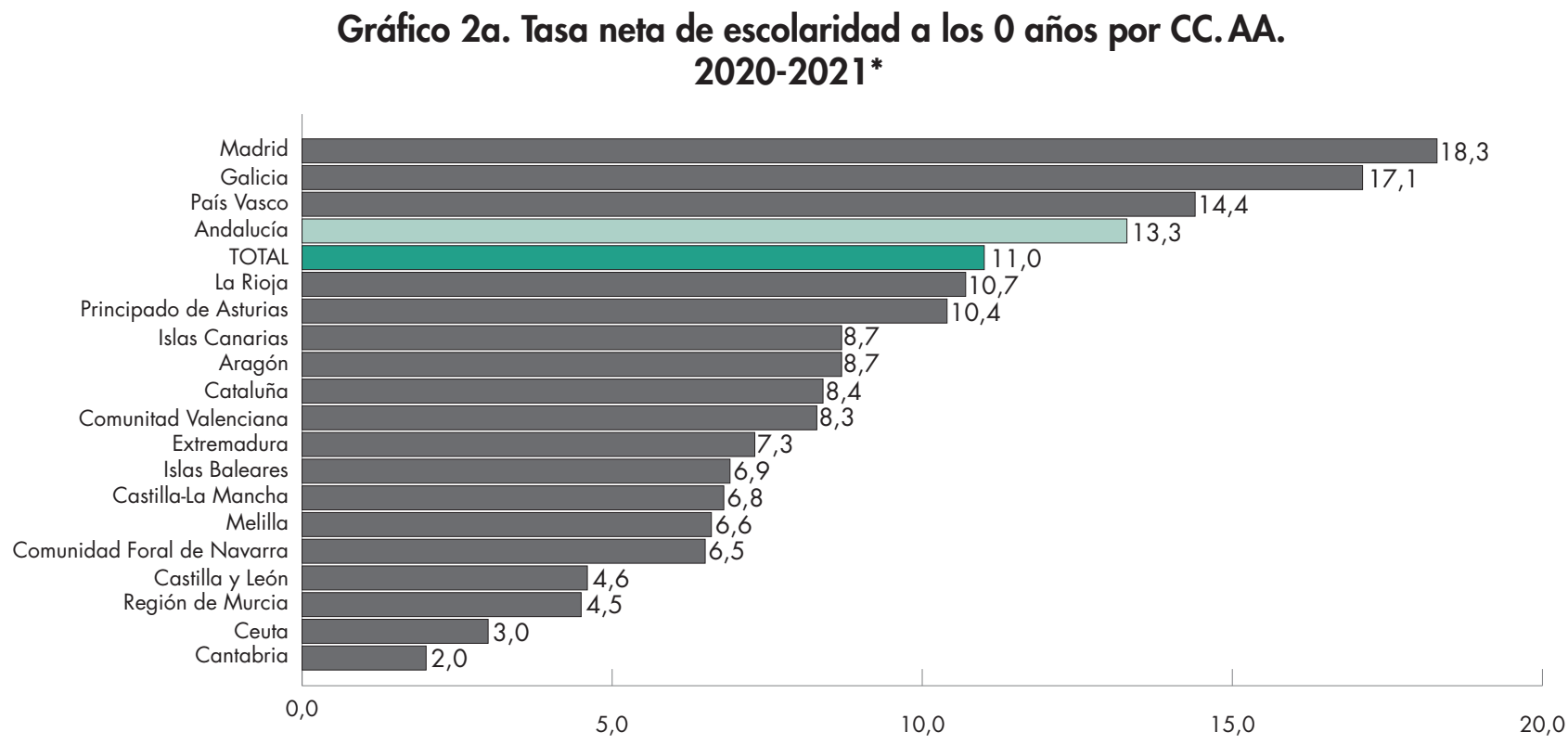

Fuente: Ministerio de Educación y Formación Profesional. Las cifras de la educación en España.

*Los datos de este curso son Datos Avance.

De forma similar, los gráficos $2 \mathrm{~b}$ y 2c nos muestran cómo las tasas de escolarización andaluza a las edades de 1 y 2 años se sitúan claramente por encima de las medias de España. Y no muy por debajo de comunidades autónomas más ricas y con mucho menos desempleo, como Madrid y País Vasco. Finalmente, en los gráficos citados pueden apreciarse las llamativas diferencias en tasas de escolarización por comunidades autónomas que se dan en el mosaico de la EI-03 estatal.

\section{Gráfico 2b. Tasa neta de escolaridad a 1 año por CC. AA. 2020-202 1*}

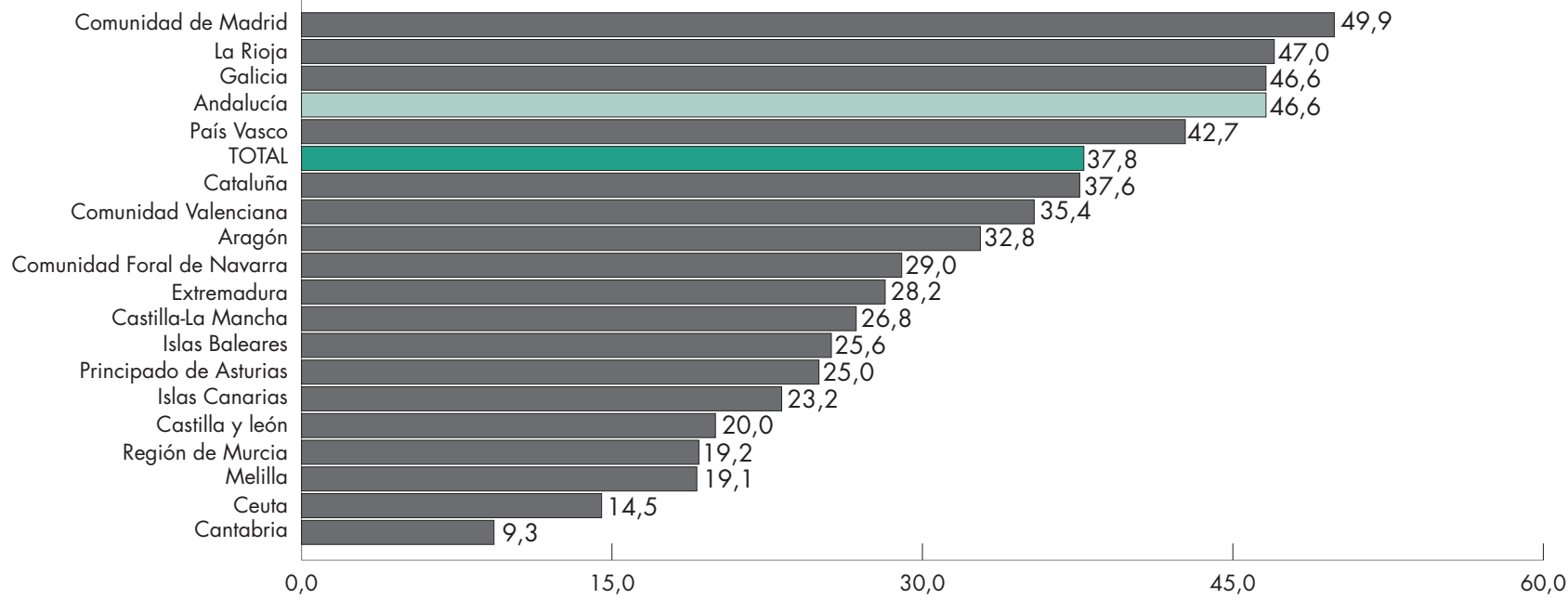

Fuente: Ministerio de Educación y Formación Profesional. Las cifras de la educación en España.

*Los datos de este curso son Datos Avance. 


\section{Gráfico 2c. Tasa neta de escolaridad a los 2 años por CC.AA.} 2020-2021*

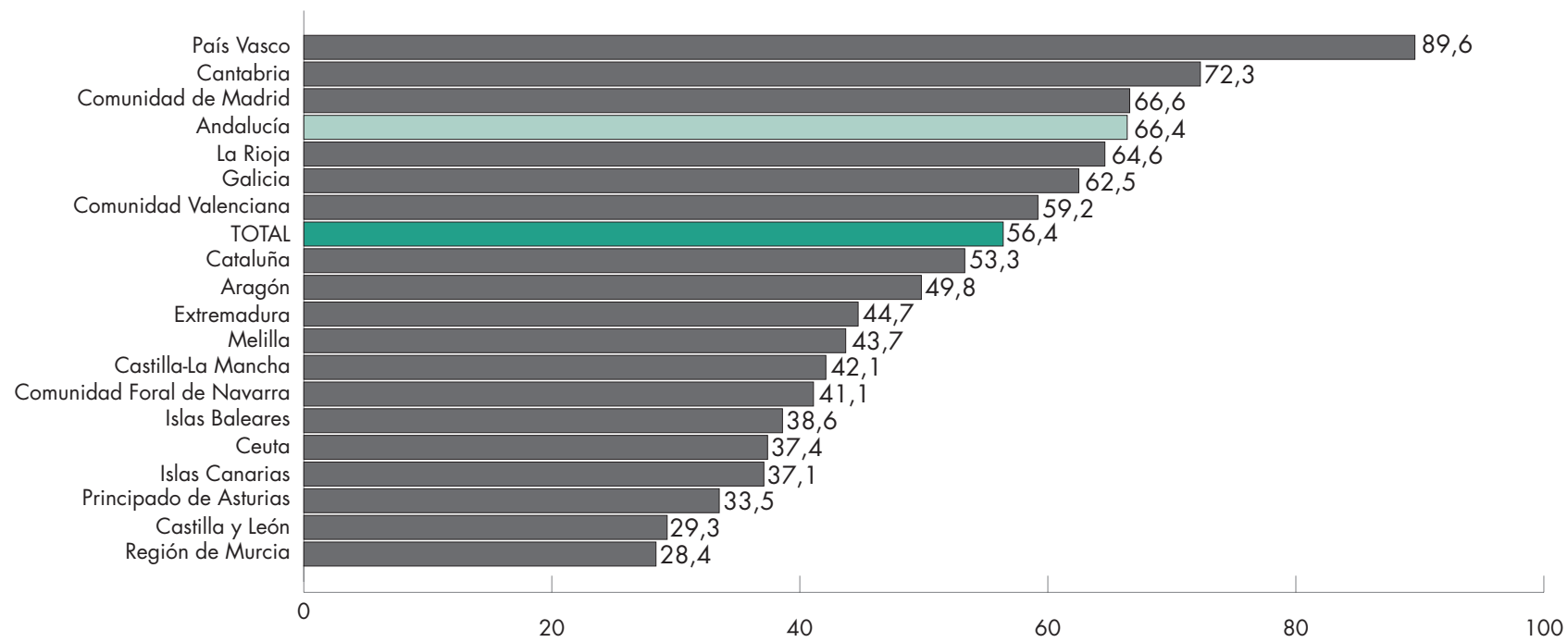

Fuente: Ministerio de Educación y Formación Profesional. Las cifras de la educación en España.

*Los datos de este curso son Datos Avance.

Por tanto, la expansión de la EI-03 andaluza es en gran medida consecuencia de una política pública que captó en su momento las necesidades y expectativas de las familias. Como revela un estudio del CIS (2009), cuyos datos hemos explotado para Andalucía ${ }^{11}$, la creación de más escuelas infantiles ya figuraba en aquellos años como la principal demanda «para una buena Educación Infantil», de forma más intensa que en la media nacional.

\section{Gráfico 3. Medidas de la administración que deben ser prioritarias para una buena Educación Infantil. Comparación Andalucía y España}

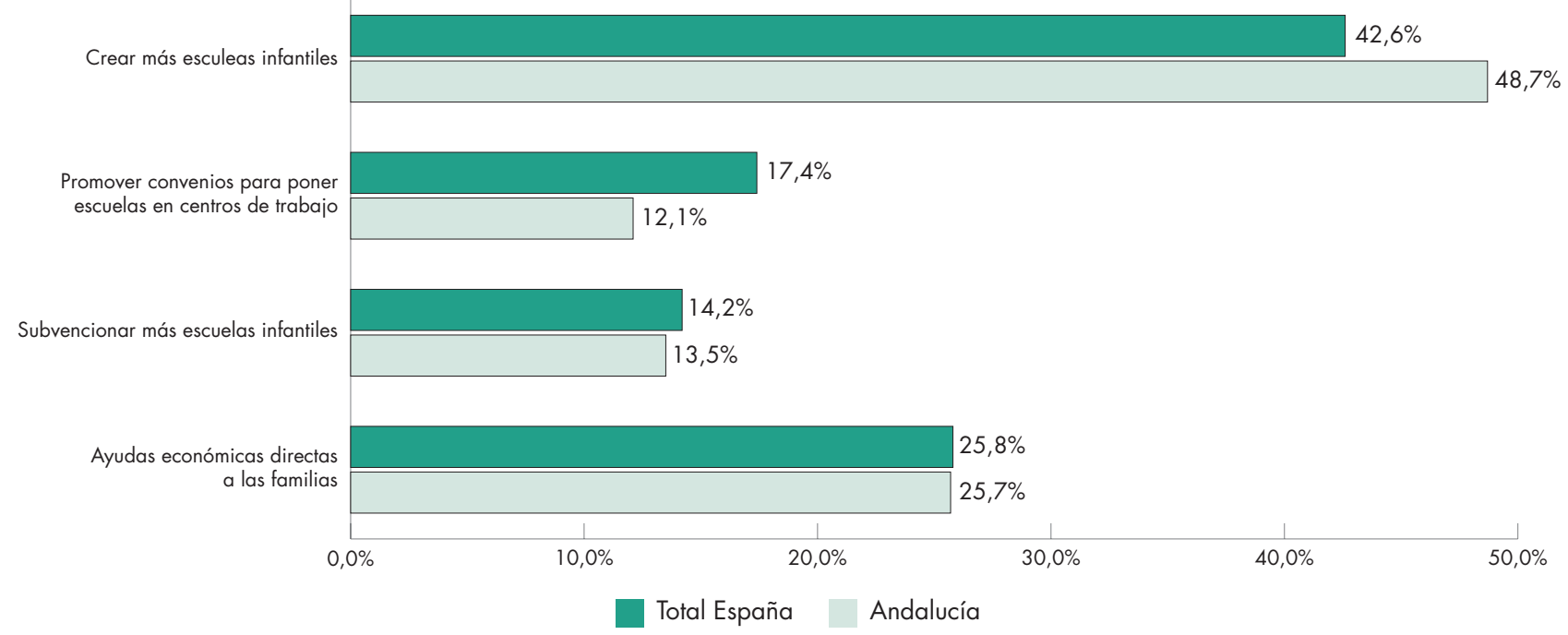

Fuente: elaboración propia. Microdatos del CIS. Estudio 2788. Barómetro febrero 2009.

11 Estudio de ámbito nacional realizado en febrero de 2009 con muestra global de 2.500 encuestas, 439 corresponden a Andalucía. Para un nivel de confianza del $95,5 \%$ (dos sigmas), y P $=$ Q, el error real para el conjunto de la muestra y en el supuesto de muestreo aleatorio simple es de $\pm 2,0 \%$ para la población española. Gabaldón y Täht (2019) también han utilizado recientemente esta encuesta, sin continuidad desde 2009, para identificar actitudes hacia los servicios de atención a la infancia en función de la clase social y la ideología política. 
Además, es relevante destacar que esto era extensivo a todos los estratos educativos de la sociedad andaluza. Especialmente entre las personas con menor capital escolar, que perciben que el aumento de la oferta de escuelas infantiles (52,6\%) es aún más importante que las ayudas directas (29,8\%).

\section{Gráfico 4. Medidas de la administración que deben ser prioritarias para una buena Educación Infantil según nivel de estudios. Andalucía}

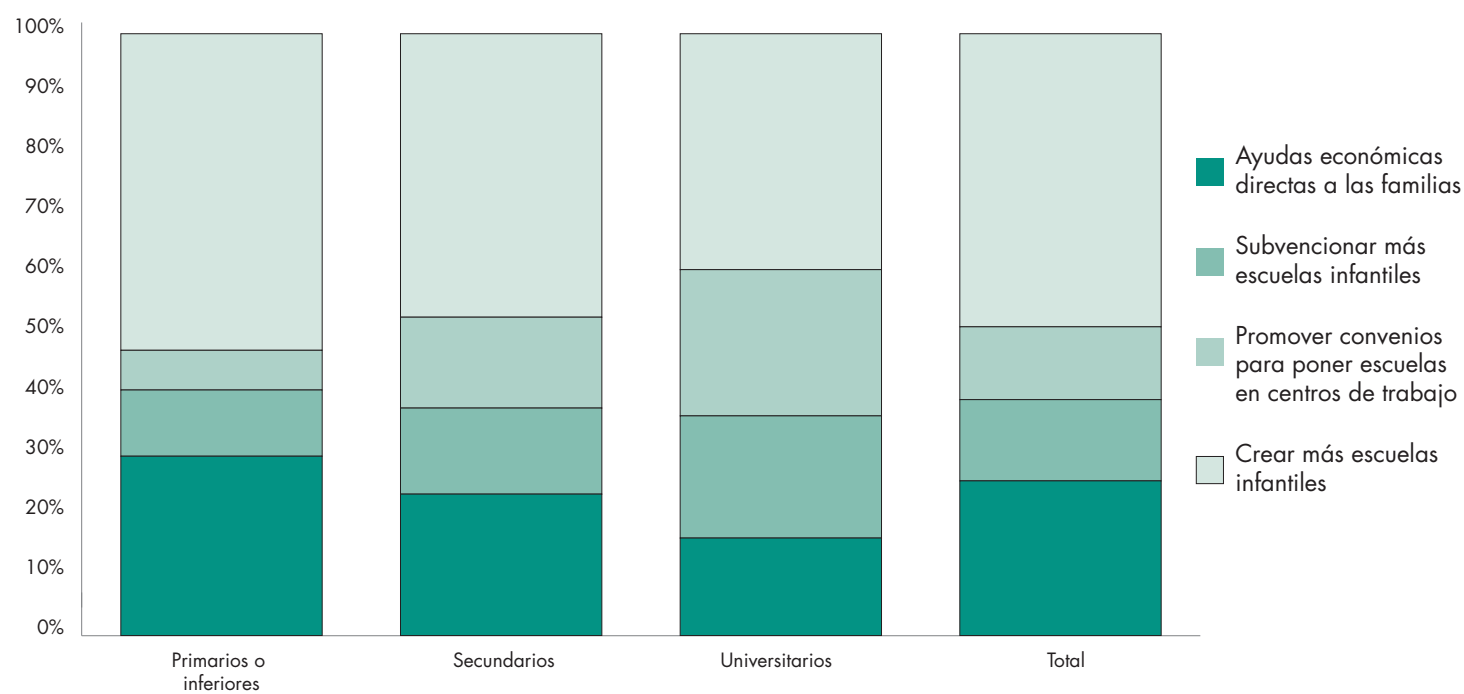

Fuente: elaboración propia. Microdatos del CIS. Estudio 2788. Barómetro febrero 2009.

Por parte de la administración andaluza se consiguió multiplicar las plazas de EI-03, paliando en gran medida dos de los grandes problemas que arrastraba la EI-03 andaluza hace quince años: el déficit (entonces) de plazas y los desequilibrios territoriales en la oferta de éstas. Sin embargo, en comparación con el incremento que alcanzará la red privada de EI-03 que se incentivó, se incentivó y creció mucho menos (y solo en los primeros años) la red pública. Las profesionales y directivas más experimentadas de la EI-03 privada reconstruyen así cómo se implantó el modelo hoy vigente de servicios en EI-03, desglosado entre «atención socioeducativa» y «comedon»:

"Es que te venian y te decian: "monta un centro alli por favor, que no tengo plazas". La Delegación de Sevilla fue tremendo $[\ldots]$, machacó para que montáramos determinados centros y en determinados sitios, o sea nos animó, no tenían para dar cobertura [...] Sabiamos la red de plazas que babia y sabiamos la red de plazas que habian prometido. Y nosotros deciamos: ¿esto cómo lo van a hacer, si es que esto no bay? Y ahora empezaron a promover, que biciéramos nuestra inversión privada, que nosotras somos maestras, nosotras no somos empresarias». (REPREPATRONAL.Sev).

Los efectos de la apuesta y la acción política privatizadora en materia de EI-03 podemos discernirlos en el Gráfico 4. Muestra la evolución de la distribución del alumnado de EI-03 por tipos de centros. Da cuenta de cómo se impone claramente la expansión de la oferta privada en detrimento de las escuelas infantiles públicas. Estas últimas además pasaron a funcionar bajo el mismo modelo que los centros adheridos basados, por un lado, en bonificaciones graduales a la matriculación, hasta un 100\%, a un sector de las familias de menor renta. Por otro lado, basados en la tasación administrativa del coste de los servicios a pagar por las familias en todos los centros privados que decidieron adherirse a las condiciones de la Junta de Andalucía, la gran mayoría: 


\section{Gráfico 5. Alumnado de 0-2 años matriculado por tipo de centro. Datos absolutos. Andalucía 1999-2021}

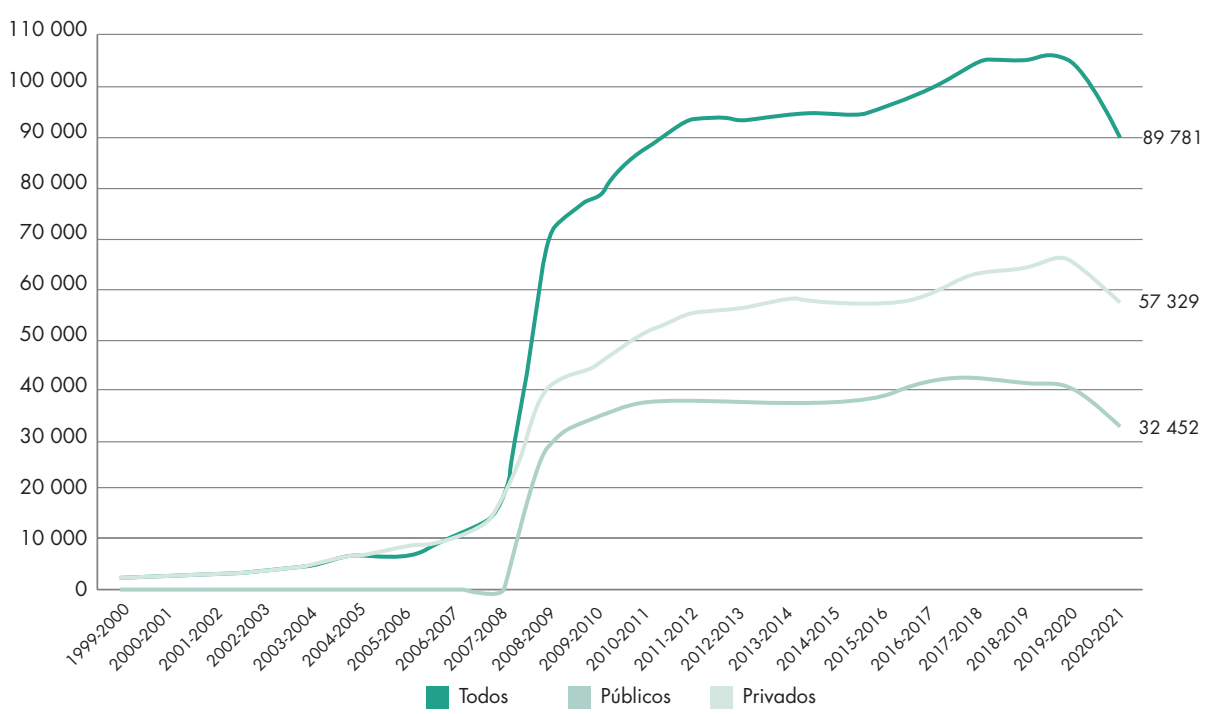

Fuente: elaboración propia a partir de datos del Ministerio de Educación y Formación Profesional.

Los últimos datos para el curso 2019-2020, recogidos en la Tabla V y facilitados por la propia Consejería de Educación y Deportes, muestran el peso dominante de la oferta privada en el primer ciclo de infantil. Todo lo contrario a lo que ocurre en el segundo.

Tabla V. Distribución del alumnado de infantil según ciclo y titularidad del centro. Andalucía. Curso 2019-2020

\begin{tabular}{lcccc}
\hline & PÚBLICO & PRIVADO & PRIVADO NO & TOTAL \\
& CONCERTADO & CONCERTADO & $100,0 \%$ \\
\hline Primer ciclo (1) & $40,5 \%$ & $54,0 \%$ & $5,5 \%$ & $100,0 \%$ \\
\hline Segundo ciclo & $76,9 \%$ & $20,4 \%$ & $2,7 \%$ & \\
\hline
\end{tabular}

Fuente: Consejería de Educación y Deporte - Junta de Andalucía (2020).

En consecuencia, luces en el grado de satisfacción de la demanda poblacional: la administración responde creando las plazas que se necesitaban. Sombras, en cambio, en el modelo adoptado para cubrir las plazas. No es neutral el modelo privatizador adoptado con bonificaciones a familias de muy escasa renta y tasación de precios para las familias no bonificadas. Este modelo tiene varias consecuencias. Así, como revela el mucho mayor coste real de las plazas en centros públicos de EI-03, la privatización con bonificaciones a familias de muy bajos ingresos y precios tasados para las demás no se ajusta a los costes reales de los servicios. Ello ahoga económicamente a las empresarias del sector y promueve la precarización de los servicios ofrecidos. Como veremos más detenidamente más adelante, el modelo vigente afecta a la calidad del sistema:

«No cabe duda de que el aumento de oferta y la respuesta a la demanda, en los últimos años, ha sido muy elevada. Otra cosa es cómo se está haciendo, desde el punto de vista más educativo [...] La generalización de la escolarización en 0-3 se está produciendo. Ahora: ¿la atención de estos niños está siendo todo lo educativa que debería de ser? Desde mi punto de vista, no: mantiene un fuerte componente asistencial. Yo creo que el salto cualitativo que habría que dar, unido a la generalización casi total, 
es el de mayor calidad educativa en la atención de estos niños [...] En los últimos años, ocho, nueve o diez años, el incremento de plazasy de atención ha sido tremendo, porque ha habido una demanda, no por otro motivo. Pero no se ha dotado de toda la calidad deseable». (INSPEDU1.Sev).

En la EI-03 andaluza también puede hablarse de déficits en otras ayudas a la escolarización diferentes a las bonificaciones previstas. En principio, Andalucía sería de las comunidades en las que más porcentaje de familias reciben algún tipo de ayuda para Educación Infantil. Sin embargo, hay un problema de fuentes que no desagregan por ciclos. En realidad, todas las ayudas andaluzas más allá de las bonificaciones establecidas para las EI-03 recaen en familias con menores en segundo ciclo de infantil. Además, aunque la cobertura sea algo más amplia en dicho ciclo, el monto es inferior en Andalucía al de otras comunidades (Valdés et al., 2021: 76-77).

\subsection{Consecuencias de la apuesta privatizadora: familias autoexcluidas por moti- vos económicos}

Estudios europeos muestran que los servicios de atención a la infancia no suelen estar dirigidos a familias de bajos ingresos (Bonoli et al., 2017; Pavolini y Lancker, 2018). Las familias españolas son las que más gastan en servicios de EI-03 en el contexto europeo (Vandenbroeck, et al., 2018; Save The Children, 2019). Son las familias con mayores ingresos y nivel de estudios las que más y más tempranamente recurren a la EI-03 (Abrassart y Bonoli, 2015; Río Ruiz y Martín Gimeno, 2017). Más del $50 \%$ de las familias que declaran querer matricular en EI-03 lo descarta por motivos económicos. Solo para una de cada cuatro familias la EI-03 resulta completamente gratuita (Save The Children, 2019; Castellanos y Perondi, 2018; Andres-Candelas y Rogero-García, 2019).

Los planteamientos de universalización del ciclo parten de la idea de reducir las barreras de acceso al mismo, siendo los condicionantes económicos el foco principal de las medidas que se llevan a cabo, como las bonificaciones a las familias. La reducción del gasto público educativo que hemos experimentado ha bloqueado, no obstante, que las distintas medidas autonómicas de promoción y bonificación de la EI-03 de la última década, por lo general mal financiadas, hayan sido eficaces para reducir la desigualdad de acceso (Bonal y Scandurra, 2019).

¿Y en el caso andaluz? ¿Qué papel juegan las barreras económicas? ¿Qué se ha hecho y qué se ha dejado de hacer en materia de ayudas a las familias con el objetivo de favorecer la escolarización temprana? ¿Las barreras económicas y las insuficiencias de las ayudas a la escolarización temprana afectan más a familias en situaciones de pobreza severa, o a otras familias trabajadoras cuya situación de pobreza es más relativa?

Andalucía tiene niveles de renta por debajo de la media española ${ }^{12}$ y mayores tasas de riesgo de pobreza ${ }^{13}$. Esta realidad, como hemos visto, se combina con unos altos porcentajes de matriculación en EI-03. En este escenario, Andalucía ofrece desde 2009 un Plan de Ayudas a las familias más desfavorecidas (las bonificaciones) para poder optar a una plaza gratuita o ampliamente subvencionada según criterios establecidos por el IPREM (Indicador Público de Renta de Efectos Múltiples).

12 La renta media neta por hogar en España en 2019 era de $30690 €$; en Andalucía descendía $25909 €$. Fuente: INE. Encuesta de Condiciones de Vida 2020. https:// www.ine.es/jaxiT3/Tabla.htm?t=9949\&L=0.

13 La Tasa de Riesgo de Pobreza en España en 2019 era del 21 \%; en Andalucía ascendía al 28,5\%. Fuente: INE. Encuesta de Condiciones de Vida 2020. https:// www.ine.es $/$ jaxiT3/Tabla.htm?t=9963\&L=0. 


\section{Tabla VI. Resumen de bonificaciones aplicables en El-03 y distribución.} Curso 2017/2018 ${ }^{14}$

\begin{tabular}{|c|c|}
\hline INGRESOS Y CIRCUSNTANCIAS DE LA UNIDAD FAMILIAR & BONIFICACIÓN \\
\hline Circunstancias sociofamiliares que originen la adopción de medidas de protección del o de la menor & \multirow{6}{*}{$100 \%$} \\
\hline Circunstancias sociofamiliares que originen carencias o dificultades en la atención a las necesidades básicas & \\
\hline Hijos o hijas de mujeres atendidas en los centros de acogida para mujeres víctimas de la violencia de género & \\
\hline Victimas de terrorismo o sus hijos o hijas & \\
\hline Renta Per Cápita inferior al 0,5 IPREM (37598€) & \\
\hline Renta Per Cápita de Familias Monoparentales inferior al 0’75 IPREM $(5639,69 €)$ & \\
\hline Renta Per Cápita igual o superior al 0,5 e inferior o igual al 0,6 IPREM $(3759,8 €-4511,75 €)$ & $80 \%$ \\
\hline Renta Per Cápita superior a 0,6 IPREM e inferior o igual a 0,75 IPREM (4511,75€ - $(5639,69 €)$ & $70 \%$ \\
\hline Renta Per Cápita superior a 0,75IPREM e inferior o igual a 0,9 IPREM (5639,69€- 6767,63€) & $60 \%$ \\
\hline Renta Per Cápita superior a 0,9 IPREM e inferior o igual a 1,0 IPREM (6767,63€-7519,59€) & $50 \%$ \\
\hline Renta Per Cápita superior a 1,0 IPREM e inferior o igual a 1,1 IPREM (7519,59€ - $8271,55 €)$ & $40 \%$ \\
\hline Renta Per Cápita superior a 1,1 IPREM e inferior o igual a 1,2 IPREM (8271,55€-9023,51 €) & $30 \%$ \\
\hline Renta Per Cópita superior a 1,2 IPREM e inferior o igual a 1,3 IPREM (9023,51 €-9775,47€) & $20 \%$ \\
\hline Renta Per Cápita superior a 1,3 IPREM e inferior o igual a 1,4 IPREM (9775,47€ - 10527,43€) & $15 \%$ \\
\hline Renta Per Cápita superior a 1,3 IPREM e inferior o igual a 1,4 IPREM (9775,47€- 10527,43€) & $10 \%$ \\
\hline Renta Per Cópita superior a 15 IPREM (11 279,39€) & $0 \%$ \\
\hline
\end{tabular}

Fuente: Consejería de Educación y Deporte - Junta de Andalucía (2020).

¿Qué alcance tiene este programa de bonificaciones? Hay que tener en cuenta que buena parte de las familias andaluzas que se ven excluidas de las bonificaciones no limitarán su matriculación al periodo ordinario, el único estipulado para poder solicitar las ayudas. Lo hacen a lo largo del año. Muchas otras familias, aunque también matricularán en el periodo ordinario, no solicitarán en ese momento bonificaciones porque saben que sobrepasan los umbrales de renta fijados para ello. Falta por tanto conocer del conjunto de familias matriculadas en cualquier momento del año, en periodo ordinario o fuera, qué porcentaje alcanzó bonificación total o parcial. Tenemos los datos, en cambio, de quienes al matricular en el periodo ordinario solicitaron las bonificaciones (Tabla VII). En el curso 2019-2020, casi un $50 \%$ de familias que solicitaron plaza y bonificación — casi siempre informadas e incentivadas de sus posibilidades por las directoras de los centros- consiguieron la gratuidad total del servicio. La bonificación media fue de un 71,69\%. El 8,54\% de familias que la solicitaron ese año, dentro del periodo ordinario, no recibieron ninguna bonificación y el 47,5\% recibió la bonificación total. 


\title{
Tabla VII. Bonificaciones según tramos para matriculados en periodos de matriculación oficial
} en primer ciclo de Educación Infantil. Curso 2019-2020

\begin{tabular}{ccc}
\hline TRAMOS DE BONIFICACIÓN & $\begin{array}{c}\text { ALUMNADOS MATRICULADOS } \\
\text { SEGÚN TRAMO DE BONIFICACIÓN } \\
\text { QUE RECIBEN }\end{array}$ & PORCENTAJE \\
\hline 100 & 46.505 & $47,57 \%$ \\
\hline 80 & 7.863 & $8,04 \%$ \\
\hline 70 & 10.112 & $10,34 \%$ \\
\hline 60 & 7.827 & $8,01 \%$ \\
\hline 50 & 4.282 & $4,38 \%$ \\
\hline 40 & 3.732 & $3,82 \%$ \\
\hline 30 & 3.235 & $3,31 \%$ \\
\hline 20 & 2.338 & $2,39 \%$ \\
\hline 15 & 1.929 & $1,97 \%$ \\
\hline 0 & 1.595 & $1,63 \%$ \\
\hline TOTAL & 8.352 & $8,54 \%$ \\
\hline
\end{tabular}

Fuente: Datos proporcionados Consejería de Educación de la Junta de Andalucía, por petición específica.

Nota: estos menores corresponden con aquellos que presentaron solicitud de plaza en periodo de matriculación ordinario y que son, junto con los menores matriculados a instancias de Servicios Sociales en cualquier momento del año, quienes pueden percibir ayudas-bonificaciones.

La pregunta que cabe hacerse es, atendiendo a estos datos, cómo se reparte el gasto total entre las familias y la Administración en la enseñanza EI-03 en Andalucía. Si se toman como referencia los tramos de bonificación y el número de matrículas en cada tramo de la Tabla VII, y el precio medio de la plaza que era de 220,72€ en el curso 2019-2020, se puede estimar que las familias asumen el 28,3\% del coste total de los servicios que les implican a ellos directamente. Esto puede convertirse en una carga excesiva para muchas de las familias que tienen una situación precaria y que no entran dentro de los tramos altos de bonificaciones ${ }^{15}$.

Por tanto, atendiendo a los umbrales de renta fijados, puede concluirse que en Andalucía los grupos poblacionales más vulnerables económicamente son los más claramente beneficiados de las bonificaciones de la EI-03. No hay, por tanto, datos para afirmar que las barreras económicas (el coste de la plaza de $278,88^{16}$ euros) sean decisivas en la menor matriculación y la mayor lejanía de las familias andaluzas más vulnerables de la EI-03.

\begin{abstract}
«¿Colectivos más desfavorecidos? Entendemos que tal cual está montado actualmente el sistema de ayuda que van dirigidas a las familias, precisamente son estos los colectivos que más pueden beneficiarse de ese acceso al primer ciclo, ya que, tanto por niveles de renta como por situaciones de grave riesgo, tienen garantizada la gratuidad al $100 \%$ de la escolarización. Entendemos que no estamos más que favoreciendo o fomentando que este alumnado pueda estar escolarizado en las mejores garantías». (TÉCNI1.Junta.Sev).
\end{abstract}

16 Incluye «atención socioeducativa» (209 euros) y «comedon» (69 euros) opcional. El servicio «taller de juego» (55 euros) puede pagarse por día, funciona como servicio de ampliación de horarios, y son pocos los centros que lo ofrecen. En 2020, tras la realización del trabajo de campo, la bonificación recibida por los centros se actualizó hasta 320 euros. Para las familias sin bonificación se mantuvo el precio. Los pocos centros privados no adheridos al Plan de la Junta de Andalucía tienen libertad de determinación de precios, pero para los centros públicos y privados adheridos estos eran los precios fijados a cobrar. También persisten aportaciones de los padres para sufragar actividades puntuales. 
Hay barreras económicas, pero no precisamente para las familias más pobres. En vez de por motivos económicos, el alejamiento de estos perfiles de la EI-03 debe explicarse principalmente por factores culturales y de género, como el que representan las ideologías de la maternidad tradicional (Johnston y Swanson, 2006) vigilada entre redes de interconocimiento denso:

«En los sectores más desfavorecidos y con menos nivel cultural, nos siguen viendo como asistencial, y entonces es el padre el que le dice a la madre, pero si tú no estás trabajando para qué vas a llevar al niño a la guardería, para qué, para sentarte tú todo el día a tomar café con las amigas en el bar». (DIRE5.Privada.Hue).

«Suelen ser madres muy jovencitas con media de dos o tres niños [...] Me han confesado "mi marido me ha dicho que no, que es muy chico y qué voy a hacer yo"》. (DIRE1.Privada.Sev)

Frente a los sectores más pobres, cuyo alejamiento de la EI-03 no puede explicarse principalmente por barreras económicas, al menos en Andalucía, los estrictos criterios del IPREM utilizados para bonificar dejan al margen de dichas bonificaciones a las muchas más familias precarias estabilizadas. Familias con una renta per cápita de 11500 euros (1,5 superior al IPREM) quedan fuera de cualquier posibilidad de subvención ${ }^{17}$. Muchas familias «mileuristas» interesadas desisten por motivos o cálculos económicos coste-beneficio:

"Porque abora mismo estamos solamente en un $47 \%$ de la escolarización, el resto de los niños no se escolariza, la gran mayoría porque no pueden pagarlo. Es que los mileuristas siguen quedando fuera de la ayuda, eh [...] Es que los mileuristas no tienen ayuda, una familia de dos miembros de unidad familiar mileuristas no tiene ayuda [...] Si claro, bay una familia que le entraban 11279, ya no tenía opción a ayuda" [...] Trabajan los dos, pero bueno muchas veces no te escolarizan y se los dejan a los abuelos eh, yo tengo muchos mileuristas que no pueden, es que hay que ayudar a los mileuristas (C)». (REPREPATRONAL.Sev).

"Cuando vienen, a partir del mes que viene hacemos la reserva de plazas, ya te digo que cuando vienen una de las cosas que yo trato de dilatary de enfriar un poco es el "cuánto me va a salir" [...] Siempre trato de enfriar eso porque muchas personas llegan y en el momento que saben lo que tienen que pagar, y saben que sí hay muchas plazas, automáticamente descartan y yo trato de acoger, porque no estamos en disposición». (DIRE2.Pública.Sev).

$\mathrm{Al}$ evaluar el peso de los factores económicos sobre la EI-03 andaluza de nuevo aparecen luces y sombras. Las familias pobres generalmente con bajo capital educativo se podrían beneficiar ampliamente de plazas gratuitas, aunque lo hacen en menor medida debido a barreras culturales y de género. Las familias de clases populares, «precarias estabilizadas», quedan generalmente fuera de las ayudas-bonificaciones ${ }^{18}$. Esto condiciona negativamente, desincentiva y aplaza la escolarización.

\footnotetext{
17 La propia Viceconsejera actual de Educación de Andalucía reconocía a finales de 2021 que: «hay un 52\% [de alumnado] que no llega a escolarizarse porque no puede pagar un importe significativo para cualquier economía familian», señalando además que «esto está suponiendo un freno importante a la escolarización en esta etapa». Véase Eldiario.es, Ed. And. 16/12/2021. Disponible: https://www.eldiario.es/andalucia/andalucia-rectifica-acepta-fondos-europeos-obligan-crear-4-000-plazas-guarderia-publicas-gratis-12-meses_1_8580162.html.
}

18 Bajo esta expresión para el caso de Andalucía designaríamos a perfiles de hogares que dependen de salarios generadores de ingresos regulares y que permiten 
Hay además condicionantes administrativos a la hora de distribuir las bonificaciones que incrementan la dificultad económica a la hora de matricular, así como de proyectarse con seguridad económica en el ciclo. En primer lugar, y como también muestra la exclusión de muchos hogares del Ingreso Mínimo Vital, al depender las bonificaciones de la renta familiar de dos años antes muchas familias que atraviesan dificultades económicas en el momento de matricular se ven sin embargo excluidas, por aquellas rentas antiguas, de la posibilidad de las bonificaciones que necesitarían y tendrían atendiendo a los ingresos del momento de la matriculación. En segundo lugar, aunque la oferta de plazas es cada vez más amplia y los casos son infrecuentes, en situaciones puntuales de desequilibrio entre oferta y demanda superior de plazas en un lugar, la normativa otorga al trabajo asalariado de la pareja preferencia materializada en puntos por esta condición. Ello en detrimento de los hogares con cónyuges en desempleo. Normativamente se privilegia y reproduce así una visión anticuada de la EI-03 como mero servicio de conciliación. En casos de escasez de plazas, sobre todo se dificultaría el acceso a la EI-03 a los perfiles de hogares que reúnen frecuentemente a los perfiles de menores a quienes, según los expertos, más críticamente beneficiaría una escolarización temprana (Cebolla-Boado et al., 2014).

\subsection{Condiciones de escolarización y calidad en la Educación Infantil 0-3 de Anda- lucía: consecuencias de la infrafinanciación}

No basta con la escolarización temprana. Especialmente para los colectivos más vulnerables, la eficacia pedagógica de la EI-03 descansa en la calidad del sistema (Melhuish et al., 2015; Schleicher, 2019). El principal condicionante de la calidad en la EI-03 andaluza es su infrafinanciación. Las generalizaciones resultan odiosas. Hay diversidad de condiciones de escolarización también entre la oferta privada adherida de EI-03, hemos visto que la dominante y la que más se ha expandido en Andalucía. No obstante, a pesar de la diversidad de empeños y apuestas empresariales, la calidad de estos centros se resiente regularmente por la insuficiente financiación que reciben por i) familias bonificadas, así como ii) por el precio regulado administrativamente por plaza (278 euros $\left.{ }^{19}\right)$ que deben cobrar a las no bonificadas. La patronal de la EI-03 acostumbra a rechazar que subsistan diferencias de calidad entre centros públicos y privados-adheridos. Basta, en cambio, indicar un dato que el sector utiliza para reclamar más financiación para sus centros infrafinanciados, cada vez con más plazas vacantes. En EI-03 públicas el precio-coste real estimado por plaza es tres veces mayor que en las privadas:

"¿Qué tampoco hemos encontrado? Pues un reconocimiento económico. Llevamos doce años y el coste plaza de un niño en su centro está tres veces por encima a lo que a nosotras nos están pagando, claro... En aspectos económicos a lo mejor no quieren que lo toquemos, pero... [...] Ellos mismos [desde la Consejería] hicieron este estudio paralelamente, llegamos a la misma conclusión: que el coste de la plaza de infantil es muy costoso...». (DIRE5.Privada.Hue).

"Elprecio plaza de un centro, el coste plaza de un niño en una propia de la Junta es tres veces el nuestro, eh, de aqui de Andalucía [...] Estamos haciendo un gran servicio a la Consejería. Les salimos muy baratas». (REPREPATRONAL.Sev).

superar — frecuentemente por estrecho margen— el umbral de la pobreza. Bajo este umbral se situaría, según la Encuesta de Condiciones de Vida 2020, el 28,5\% de hogares (21\% en España). Casi tres de cada diez hogares andaluces que podrían disfrutar, en cambio, de bonificaciones completas en EI-03.

19 La bonificación que pueden recibir los centros por plaza se actualizó en 2020 hasta 320 euros, aunque todas las partes reconocen que sigue siendo insuficiente. El precio de las matrículas no bonificadas no se incrementó. 
La diferencia de costes de mantenimiento entre plazas públicas y privadas explicará, en gran medida, las generalizadas diferencias de calidad entre escuelas infantiles públicas y privadas. Aunque al establecerse en ambas el mismo sistema de bonificaciones y precios se promueve la confusión de muchas familias que no discriminan suficientemente entre plazas públicas y privadas a la hora de elegir, haciéndolo muchas veces por la cercanía entre centros muy disímiles al mismo precio, las escuelas infantiles públicas disponibles al mismo precio y bajo las mismas bonificaciones reúnen regularmente mayor calidad que las privadas. Para una Educación Infantil de calidad es insuficiente la financiación derivada de contar con familias bonificadas en los centros. Y es insuficiente el coste por plaza que se cobra a las familias no bonificadas. Toda esta precariedad se traduce además en demandas de flexibilización — cuando no en transgresiones - de las normativas de escolarización fijadas para el ciclo, como las ratios por aula en función de la edad, o la separación de menores por edades.

«Hay problema de gestión económica... Están haciendo un esfuerzo muy grande, se mueven con unos márgenes de incertidumbre muy amplios... Asi tampoco se puede gestionar bien [...] ¿Qué hacen? Pues se centran más en gestionar con pocos recursos para dar el mejor servicio posible, pero claro, al final eso nos aboca a lo asistencial». (INSPEDU1.Sev).

«No tiene color. La privada, en mis años qué te voy a contar, pero hoy día que también pregunto y me muevo, no deja de ser un negocio [...] Las ratios son mucho más numerosas que las nuestras, el personal es un personal explotado, donde la tutora de una clase pues igual te limpia que te atiende [...] Hay madres que dicen: "bueno como es una guardería que yo lo que quiero es guardar a mi bijo y la tengo aqui debajo y encima me quitan el 15\% aqui también, pues lo dejo aqui». (DIRE4. Pública-Junta.Sev).

La infrafinanciación condiciona negativamente diversas facetas claves de la calidad educativa ${ }^{20}$. En primer lugar, condicionan la calidad los bajos salarios del sector privado en todo el Estado. Escasamente por encima del salario mínimo. En una horquilla entre 13000 y 19000 euros según categorías profesionales $^{21}$. Se cobra en las distintas categorías entre un 25 y un $30 \%$ menos que en las escuelas infantiles públicas.

En segundo lugar, están los condicionantes para la formación continuada y la coordinación del personal laboral y directivo de los centros. La de la formación supone una demanda histórica del sector insuficientemente satisfecha con una tardía normativa de $2019^{22}$. Esta ofrece formalmente, pero no posibilita realmente, el acceso de las profesionales del sector a los Centros de Formación del Profesorado. Hay demandas de formación no satisfechas (García-Caballero, 2021). A su vez, la mayor parte de los centros tienen dificultades para integrar estas actividades en los mismos horarios de las trabajadoras. Para ellas el tiempo invertido en formación rara vez computa como jornada laboral. Todo ello convierte a la deseada formación continuada en la EI-03 en una quimera. De la misma manera, las condiciones de precariedad bajo las que opera en mayor medida el sector privado obstaculizan la

\footnotetext{
20 Según el informe del Defensor del Pueblo (2017), la entrada en vigor del Decreto-ley 1/2017, de 28 de marzo, generó un nuevo sistema con mejoras y ventajas respecto al anterior pero que también «discrimina» a los centros adheridos frente a las escuelas de titularidad de la Junta de Andalucía, introduciendo un importante factor de precariedad que pone en peligro la viabilidad del funcionamiento de los centros y la estabilidad laboral de los profesionales que trabajan en los mismos. 21 UGT. XII Convenio Colectivo de ámbito estatal de centros de asistencia y Educación Infantil.

22 Resolución de 12 de julio de 2019 de la Dirección General de Trabajo por la que se registra y publica el XII Convenio colectivo de centros de asistencia y Educación Infantil.
} 
profesionalización, exclusividad y eficacia de la función directiva, frecuentemente desbordada y desvirtuada al verse absorbida por cada vez más exigencias y servicios administrativos:

«La función directiva actualmente debe de tener una formación adecuada, hombre no solo puede ser maestra, tiene que contemplar otras figuras como hacen en los coles [...] En nuestra guarde no se contempla nada, solamente se contempla el director y este es el que lo tiene que hacer todo, y a veces sin formación, con lo cual pues el centro no funciona bien. Entonces yo creo que actualmente deberian de adquirir una formación especializada a la función directiva». (DIRE5.Privada.Hue).

«No tenemos margen [...] En nuestra casa, los sábados, los domingos hacemos las solicitudes [...] El convenio de subvenciones nos tiene acribilladas, porque hay que ser inspector de trabajo, inspector de hacienda [...] los problemas son para mí, si yo no tengo para pagar las nóminas los problemas son para mí, si yo no tengo para pagar la seguridad social los problemas son para mi, y te pones a mirar $y$ tú dices "me voy a ir"》. (REPREPATRONAL.Sev).

En tercer lugar, las constricciones económicas impiden la coordinación pedagógica dentro del centro, la cual ni siquiera está normativamente integrada como actividad obligatoria de ningún tipo de centro:

«El Decreto que regula la organización y funcionamiento de las escuelas infantiles, tanto públicas como privadas, en ningún momento aparece, por ningún sitio, cómo se establecerá la coordinación didáctica educativa. Por lo cual, el propio Decreto no prevé esa posibilidad, a sabiendas de que el régimen laboral de estas personas, incluidas las de titularidad pública, no contempla la posibilidad. ¿Eso tiene una incidencia en la atención educativa del alumnado? Pues no cabe duda, si no hay posibilidad de formación, si no hay posibilidad de coordinarnos, de realizar unos...». (INSPEDU1.Sev).

«Parece mentira que ya a estas alturas cómo se puede obviar ese tema tan importante. O sea, la coordinación, ya no hablamos con el segundo ciclo, sino en la misma guarde por dios, es que el personal tiene que coordinarse, tiene que reunirse, volvemos a lo mismo, falta de... Tú cómo le dices a un personal que está cobrando ochocientos, setecientos, seiscientos... que tiene que quedarse por la tarde a una reunión de equipo, es que hombre». (DIRE5.Privada.Hue).

En cuarto lugar, hay carencias generalizadas en las infraestructuras de muchas instalaciones. Este problema, aunque en menor medida, también alcanza actualmente a escuelas públicas. Estas se ven obligadas por la administración a acometer las reformas necesarias con los ingresos de matriculaciones y bonificaciones que perciben, como si fuesen centros privados. Hay desde 2009 fijadas condiciones arquitectónicas mínimas que deben cumplir los centros. Estas condiciones a veces se evalúan sobre el terreno de forma laxa. Generalmente, la adecuación de las instalaciones es valorada desde el cumplimiento de unos mínimos requisitos asistenciales, en lugar de pedagógicos:

"Hay cantidad de cosas que no cumplen la normativa [...] Si me la comparas con la concertada, siempre la pública está mejor en cuanto a distribución y espacio. Pero no te creas que cumplimos todas 
[...] Al revés, yo creo que se corre un tupido velo [...] Cuando tu exiges [...]: "oye mira es que yo tengo esta humedad", te dicen: "ah no mira eso lo cubre con la subvención como buenamente pueda"”. (DIRE4.Pública-Junta.Sev).

"Aunque muchas escuelas privadas se han autorizado, y eso requiere baber hecho una supervisión de las instalaciones, sin lugar a dudas las instalaciones de algunas escuelas infantiles dejan mucho que desear [...] Se han puesto unos requisitos minimos para un régimen asistencial, no para un régimen educativo [...] Y yo lo decía, esto no debería de haberse autorizado, pero claro [...] El informe del técnico, pues mire usted... El Decreto dice 'X' metros cuadrados, 'pun, pun', sí, ya está (...) Entonces, con lo importante que es el diseño de los edificios educativos». (INSPEDU1.Sev).

Las constricciones económicas obligan a que, tanto desde la administración, como desde los propios centros en dificultades económicas, se prime más el cumplimiento de los mínimos del proyecto asistencial que el proyecto educativo. Además, las normativas, a medida que han ido perdiendo generalidad, han ido rebajando exigencias pedagógicas para adecuarse a una realidad estructuralmente precaria donde se prima el cumplimiento de los aspectos asistenciales:

"Ha habido una implantación desigual porque una cosa ha sido el Decreto de enseñanza y la orden y el desarrollo de curriculum, y otra cosa diferente ha sido pues todas las derivaciones legislativas que ha babido en organización y funcionamiento, que a veces no se acompañan, el espiritu del currículum que recogía el BOJA con los desarrollos legales». (DIRE7.Pública-Munic.Gran).

"¿Qué observamos cuando vamos a los centros? Que los proyectos educativos son asistenciales [...] Son muy flojos, son "copia y pega", son meros trámites que se llevan a cabo porque lo tienen que tener, porque lo piden, para que el inspector lo vea. Pero luego no hay unos proyectos que respondan a un contexto, a una realidad, a un buen análisis de situación [...] En muchos casos no hay ni propuestas didácticas, no existen las propuestas didácticas que prevé el Decreto 149 [...] En muchos casos ni existen, no están elaboradas ¿ Pero, cuándo las elaboran? ¿Dónde está el horario para hacerlo y para coordinarla?». (INSPEDU1.Sev).

Los déficits también afectan a la actividad de la inspección educativa. Su labor se ha ido objetivando con el paso de los años. En la actualidad se ha pasado de solo visitar aleatoriamente algunos centros a la programación de visitas a todos los centros por parte del inspector de zona, pero hay insuficiencias en gran medida explicables porque la inspección no está especializada en Educación Infantil. Su acción abarca todos los ciclos educativos preuniversitarios (Garrido, 2021):

"La inspección educativa en 0-3 es un poco absurda. Por no decirte banal. Hay inspectores que te hablan de que, si usted ha facturado bien, ha hecho bien las facturas. Y otros, precisamente este año me be encontrado un inspector que lo que me ha pedido es lo que me tiene que pedir: cómo funciona el centro, cómo son las evaluaciones, qué proyecto educativo seguimos...». (DIRE4.Pública-Junta.Sev).

También se detecta una llamativa descoordinación entre primer y segundo ciclo de infantil, si bien la comunicación se valora como más fluida y protocolizada con los Servicios Sociales para los casos 
de menores declarados en situación de riesgo social. La nueva LOMLOE precisamente incide en la superación del déficit de coordinación detectado a la hora del tránsito entre ciclos infantiles. A escala andaluza nos consta que ha habido intentos de mejorar esta faceta, creándose grupos de trabajo. Se sigue en cambio sin un protocolo que regule el tránsito entre ciclos. La comunicación entre ambos tipos de centros, lejos de estar normalizada, se produce en situaciones excepcionales:

«Si yo sé que de aqui hay 20 niños, 10 o 11 que van al colegio que se llama Pepito Jiménez, pues ese Pepito Jiménez yo creo que debería interesarse. A lo largo de mi carrera yo creo que me han llamado dos veces de esos centros para interesarse por el niño. Pero claro, curiosamente han sido dos niños con problemas. Para el resto no me han llamado. No hay ninguna conexión, no hay nada: qué menos que preguntar, darle un informe o utilizando a los padres para que si vienen de tal centro pidan un informe para que vean en el niño sus objetivos alcanzados.. Nada. No existe nada». (DIRE4. Pública-Junta.Sev).

\section{Conclusiones}

Hace casi tres lustros que Andalucía desarrolló una arquitectura normativa de la EI-03 que regularizó las condiciones de escolarización en este ciclo y garantizó a las familias solicitantes el derecho a una plaza en él. La EI-03 hasta entonces había sido un sector poco planificado, con desequilibrios de oferta y demanda y territoriales, así como heterogéneo en precios, en servicios ofrecidos, incluso en las competencias regulatorias repartidas entre administración autonómica y municipios. Hoy en cambio Andalucía está dotada de una amplia oferta de plazas, aunque con algunos desequilibrios territoriales que afectan sobre todo a localidades de la provincia de Cádiz con déficits de oferta. Más del 90\% de las plazas disponibles, exceptuando las ofrecidas por el minoritario conjunto de escuelas infantiles privadas no concertadas, están acogidas a un sistema de bonificaciones y precios comunes.

Bajo este sistema Andalucía, hemos visto, ha acabado registrando altas tasas de matriculación en EI03. Pese a las aquí menores tasas de ocupación femenina, las tasas de escolarización en EI-03 se sitúan desde hace años entre las más elevadas de España. Estamos incluso por encima de los niveles de escolarización en este ciclo alcanzados por comunidades de mayores niveles de renta y de mayor gasto educativo en enseñanzas preuniversitarias. Hasta la tasa neta de escolarización andaluza entre 0 y 1 años se ha situado recientemente por encima de la media estatal.

La expansión ha sido indudable. Sin embargo, hemos visto, el modelo andaluz de EI-03 presenta también déficits que afectan a la igualdad de oportunidades educativas y condicionan incluso la viabilidad económica del sistema en los próximos años.

En primer lugar, estamos ante un modelo de servicios externalizados, privatizados e infrafinanciados. La historia reciente de la EI-03 en Andalucía es la de una oportunidad perdida de crecer, pero a través de la potenciación de una red pública de plazas. Por el contrario, se optó en su momento por la externalización hacia centros privados a fin de absorber la demanda poblacional y abaratar los costes de un ciclo caro, como muestra el coste real estimado de la plaza en escuelas infantiles públicas. A consecuencia de todo ello, Andalucía cuenta con una de las redes públicas de EI-03 más débiles del Estado. En cambio, este dominio de la red privada se invierte en la educación entre 3 y 6 años donde predomina claramente la oferta pública. 
Hemos visto cómo se potenció la oferta privada, incluso instrumentalizando bajo «promesas rotas» a las empresarias de la EI-03, hoy ahogadas económicamente por dos razones principalmente. La primera: la insuficiencia de los precios fijados y las bonificaciones públicas que reciben sus centros para poder prestar de manera generalizada servicios de calidad, esto es, que primen la eficacia pedagógica y superen la cobertura de mínimos asistenciales a los menores que fija la administración. Esta última, según las experiencias reconstruidas de distintos actores del sistema en distintas posiciones, tendería además a rebajar de facto las exigencias normativizadas — de infraestructuras, por ejemplo — ante el reconocimiento de la precariedad estructural e infrafinanciación que, pese a los esfuerzos de las profesionales, condiciona negativamente la calidad pedagógica de un ciclo en cada vez mayores dificultades económicas.

La segunda razón de la asfixia económica de la EI-03 andaluza es el exceso de oferta privada (en la mayoría de las zonas urbanas) en un contexto de caída de la fecundidad, acentuada con la Covid-19. Las plazas desocupadas en el conjunto de la EI-03 andaluza se estiman ya en alrededor del 30\%.

En segundo lugar, el de Andalucía es también un modelo de autoexclusiones por motivos económicos. Son los sectores de mayor nivel de renta y educacional quienes más recurren a estos servicios. Los sectores por debajo del umbral de la pobreza también pueden acceder a plazas gratuitas bonificadas al 100\%. Las barreras en estos casos serían culturales y de género. Las autoexclusiones por motivos económicos afectarían sobre todo a las familias asalariadas de bajos ingresos («los mileuristas se quedan fuera»). A familias que superan los restrictivos umbrales de renta fijados para recibir bonificaciones, pero carecen de incentivos salariales suficientes para pagar el precio de los servicios de EI-03. Todo ello redunda casi siempre en sacrificios laborales de las mujeres y en desequilibrios en las relaciones de género en los que priman los condicionantes de clase por encima de los intereses de género. Estos suelen poder defenderse y aplicarse mejor por parte de las mujeres que alcanzan posiciones de clase media profesional para poder permitirse también juzgar a las demás como reproductoras alienadas de preferencias y estrategias de maternidad tradicionales.

En tercer lugar, el andaluz es un modelo de confusiones igualitaristas entre centros públicos y privados frecuentemente desiguales en la calidad de los servicios. Al fijar el mismo sistema de precios y bonificaciones en centros privados concertados y públicos se incentiva a la población a no discriminar suficientemente entre servicios y condiciones de escolarización que, en cambio, pueden llegar a variar notablemente según titularidad del centro.

En cuarto lugar, el modelo de EI-03 de Andalucía es potencialmente conflictivo. Los actores manifiestan cierto consenso en que es necesario avanzar hacia la gratuidad del ciclo. Se está de hecho eludiendo el debate de si la gratuidad debe extenderse hasta los hogares de mayores rentas salariales: a quienes más compensa económicamente los precios actuales de los servicios escolarización temprana. La cuestión divisiva es si este avance puede hacerse, a estas alturas de privatizaciones del servicio, a través de un modelo en el que se imponga y prevalezca la oferta pública de plazas. Las diferencias y divisiones en el campo social de la EI-03, conformado por las estrategias e interdependencias de diferentes actores en creciente competencia, se concentran en la actualidad en el papel que debe jugar la oferta pública financiada por Europa frente a la oferta privada. La misma reclama también el desvío de las ayudas europeas, pero para intentar fortalecer su modelo de negocio recibiendo más alumnado bonificado y evitar la competencia de una EI-03 pública que podría próximamente verse reforzada. 
No bastará en cualquier caso con avanzar hacia la universalización y ampliación de la gratuidad del ciclo. Tras los avances realizados (y los que se esperan) en niveles de escolarización, llega el momento de mejorar las condiciones de escolarización. De mejorar la calidad del sistema frente a sus déficits señalados, en gran medida producto de una insuficiente financiación. Es el momento de la eficacia pedagógica del ciclo: de las propuestas educativas y de los mecanismos de coordinación intra e intercentros. El momento de preocuparse por las condiciones laborales en el sector privado. Por la formación continua de las profesionales, incentivándola bajo planes realistas que no descansen en la buena voluntad sin incentivos fuera del horario laboral. Además, es el momento de reevaluar si las infraestructuras de muchos centros se adecúan a los estándares de otros países de la Unión Europea.

Es quizás tarde y será difícil, porque en su momento fue incentivada la construcción de toda una red privada de escuelas infantiles. Las mismas absorben hoy miles de empleos y muchas inversiones, deudas, sueños y desvelos de mujeres maestras transformadas en empresarias. Sin embargo, en el nuevo escenario de fondos europeos para inversiones en plazas públicas, también conviene considerar los pros y contras de una posibilidad que se ha obviado en la nueva LOMLOE — quizás por su potencial conflictividad social - pese a contar con evidencias favorables: la posibilidad de estructurar toda la Educación Infantil en un único ciclo integral hasta los 6 años, como en Dinamarca.

En suma, tras los logros indudables en niveles escolarización llega el momento de reformulaciones en la EI-03 de Andalucía. La evolución de este ciclo requerirá de nuevos análisis y la materialización de nuevas líneas de investigación. En nuestra agenda está conocer las posibles conexiones y distancias sociales entre las concepciones pedagógicas legítimas de la EI-03 y las distintas percepciones y expectativas que sobre esta etapa reunirían distintas clases de familias. Conocimiento que consideramos clave para el éxito de las políticas que se apliquen en EI-03.

\section{Referencias bibliográficas}

Abrassart, Aurélien y Bonoli, Giuliano (2015): “Availability, Cost or Culture? Obstacles to Childcare Services for Low-Income Families”. Journal of Social Policy, 44 (4), 787-806. DOI: https://doi.org/10.1017/ S0047279415000288.

Andrés-Candelas, Mario y Rogero-García, Jesús (2019): "Public and Household Spending in Private, Publicly-Funded Private and Public Schools in Spain During the Economic Crisis (2007-2012)". Revista Electrónica de Investigación Educativa, 21, 1-15. DOI: https://doi.org/10.24320/redie.2019.21.e18.1756.

Bonal, Xavier y Scandurra, Rosario (2019): Equidad y educación en España. Diagnóstico y prioridades. Universidad Autónoma de Barcelona (en línea). https://www.kaidara.org/wp-content/ uploads /2020/01/Informe-Equidad-y-educaci\%C3\%B3n-en-Espa\%C3\%B1, diciembre-2019.pdf

Bonoli, Giuliano; Cantillon, Bea y Van Lancker, Wim (2017): "Social Investment and the Matthew Effect" en Anton Hemerijck (ed.): The Uses of Social Investment. Oxford: Oxford University Press. DOI: https://doi.org/10.1093/oso/9780198790488.003.0005.

Castellanos, Cristina y Perondi, Ana Carolina (2018): Diagnóstico sobre el primer ciclo de Educación Infantil en España ( 0 a 3 años). Propuesta de implantación de un sistema de Educación Infantil de calidad y cobertura universal. Estudio de viabilidad económica de la reforma propuesta y de sus impactos 
socio-económicos. Papeles de trabajo, 3 (en línea). https://www.ief.es/docs/destacados/publicaciones/papeles_trabajo/2018_03.pdf.

Cebolla-Boado, Héctor; Radl, Jonas y Salazar, Leire (2014). Aprendiraje y ciclo vital. La desigualdad de oportunidades desde la educación preescolar hasta la edad adulta. Barcelona: Fundación La Caixa.

Centro de Investigaciones Sociológicas (CIS). (2009). Estudio n. ${ }^{\circ}$ 2788. Barómetro de febrero.

Colom Ortiz, Ferrán y Gabaldón Estevan, Daniel. (2016): "Recursos educativos y primera infancia: Privatización de la educación infantil de primer ciclo en la ciudad de Valencia". Revista de Sociología de la Educación (RASE), 9 (2), 277-298.

Defensor del pueblo andaluz (2017). Educación Infantil 0-3 años. En Defensor del pueblo andaluz. Informe anual (en línea). http://www.defensordelpuebloandaluz.es/sites/default/files/informe-anual-2017/ index.php/informe-anual-completo-indice/capitulo-1-actividad-por-materias/1-4-indice/1-4-educacion/1-4-2-analisis-de-las-quejas-admitidas-a-tramite-ensenanzas-no-universitarias/1-4-2-1-educacion-infantil-anos.

Gabaldón Estevan, Daniel y Täht, Kadri. (2019): “Educación infantil, ideología y clase social. Una lectura al sur de Europa a través de los datos del CIS para España”. Revista de Sociología de la Educación (RASE), $19(3), 367-394$.

García-Caballero, Laura (2021). Condicionantes y necesidades de formación en la Educación Infantil 0-3 de Andalucía. Trabajo Fin de Grado Educación Infantil, dic. 2021, Fac. CCEE, Univ. Sevilla (mimeografiado).

Garrido, Asunción (2021). La inspección educativa en el primer ciclo de Educación Infantil. Bilbao: Rubric.

González, Amparo; León, Sandra y Moreno, Francisco Javier (2019): “Combatir la pobreza infantil en España y en Europa” en Ferran Cabo y González, Cristina (eds.): Informe social. Desigualtats davant d'un futur incert. Barcelna: Fundació Rafael Campalans.

Heckman, James J. (2011): “The Economics of Inequality: The Value of Early Childhood Education". American Educator, 35 (1), 31-35.

Ibáñez, Zyab y León, Margarita (2014): "Early Childhood Education and Care Provision in Spain" en Margarita León (ed.): The Transformation of Care in European Societies. New York: Palgrave Macmillan.

Jiménez, María, Jareño, Diana y El-Habib Draoui, Brahim. (2016): “La expansión de la educación infantil en España: entre la igualdad de oportunidades y la segregación”. Revista Iberoamericana de Educación, $72(2), 19-44$.

León, Margarita y Muñoz, Carolina (2016): "Servicios de Atención la Infancia ¿política asistencial o educativa?” en Margarita León (coord.): Empleo y maternidad. Obstáculos y desafíos a la conciliación de la vida laboraly familiar. Institut de Govern i Polítiques Públiques (en línea) https://igop.uab.cat/wp-content/ uploads/2016/01/Informe-FUNCAS-IGOP.pdf.

Mancebón, María Jesús; Ximénez de Embún, Domingo y Villar Adriano (2018): "Evaluación del efecto de la escolarización temprana sobre las habilidades cognitivas y no cognitivas de los niños de cinco/ seis años”. Hacienda pública española, 226, 123-153. DOI: https://doi.org/10.7866/HPE-RPE.18.3.5. 
Martín-Sonseca, Ángel, Rujas, Javier, Herrera, Ignacio, Ortí, Mario y Pereda, Carlos. (2016): Una aproximación al impacto social de las politicas municipales del Ayuntamiento de Madrid (2003-2015) (en línea) https:/ / www.madrid.es/UnidadWeb/Contenidos/EspecialInformativo/AuditoriaDeuda/InformesImpacto/ impactosocial.pdf.

Ministerio de Educación y Formación Profesional. (2021). Datosy Cifras. Curso escolar 2020/2021. Madrid: Gobierno de España.

Ministerio de Educación y Formación Profesional. (2021). Estadística de las Enseñanzas no universitarias. Alumnado matriculado. Curso 2020-2021. Datos Avance. Madrid: Gobierno de España.

Melhuish, Edward; Ereky-Stevens, Katharina; Petrogiannis, Konstantinos; Ariescu, Anamaria; Penderi, Efthymia; Rentzou, Konstantina; Tawell, Alice; Leseman, Paul y Broekhuisen, Martine (2015): “A Review of Research on the Effects of Early Childhood Education and Care on Child Development". CARE Project Report.

OCDE, (2021). Panorama de la educación. Indicadores de la OCDE 2021. Informe Español. Ministerio de Educación y Formación Profesional (en línea). https://www.educacionyfp.gob.es/inee/dam/jcr:3922aacd-04c045ac-b8d4-4aebb9b96ab5/panorama-2021-papel.pdf

Pavolini, Emmanuele y Van Lancker, Wim (2018): "The Matthew Effect in Childcare use: a Matter of Policies or Preferences?”. Journal of European Public Policy, 25 (6), 878-89. DOI: https://doi.org/10.10 80/13501763.2017.1401108.

Río-Ruiz, Manuel Ángel y Martín-Gimeno, Rubén (2017). Desigualdades educativas en Andalucía. En I Informe del Observatorio de Desigualdad de Andalucía (133-161). Junta de Andalucía y OXFAM Intermón (en línea). https://observatoriodesigualdadandalucia.org/recursos/i-informe-oda.

Romero-Balsas, Pedro; Rogero-García, Jesús; y Meil, Gerardo (2022). "Permisos parentales no remunerados y escuelas infantiles: ¿`son recursos de conciliación sustitutivos o complementarios?”. Revista Española de Investigaciones Sociológicas, 177, 111-126. DOI: https://doi.org/10.5477/cis/reis.177.111.

Save The Children (2016). Necesita mejorar. Por un sistema educativo que no deje a nadie atrás (en línea) https:// www.savethechildren.es/sites/default/files/imce/docs/necesita-mejorar-fracaso-escolar-savethechildren-ok.pdf.

Save The Children (2019). Donde empieza todo. Educación Infantil de 0 a 3 años para igualar oportunidades (en línea) https://www.savethechildren.es/sites/default/files/imce/donde_todo_empieza_0.pdf.

Save The Children (2021). COVID-19: Cerrar la brecha. Impacto educativo y propuestas de equidad para la desescalada (en línea) https://www.savethechildren.es/sites/default/files/2020-05/COVID19Cerrarlabrecha.pdf.

Schleicher, Andreas (2019). Helping our Youngest to Learn and Grow: Policies for Early Learning. International Summit on the Teaching Profession. Paris: OECD.

Valdés, Manuel, T.; Sancho Gargallo, Miguel Ángel y De Esteban Villar, Mercedes (2021). Indicadores Comentados sobre el estado del sistema educativo español. Madrid: Fundación Ramón Areces y Fundación Europea Sociedad y Educación. 
Van Belle, Janna. (2016). Early Childhood Education and Care (ECEC) and its Long-term Effects on Educational and Labour Market Outcomes. Santa Monica: RAND.

Van Huizen, Thomas; Dumhs, Lisa y Plantenga, Janneke (2016): “A Cost-Benefit Analysis of Universal Preschool Education. Evidence from a Spanish Reform”. USE Discussion Paper Series (16-11), 1-29.

Vandenbroeck, Michel; Lenaerts, Karolien y Beblavý, Miroslav (2018): "Benefits of Early Childhood Education and Care and the Conditions for Obtaining Them". EENEE Analytical Report, 32, 1-87. DOI: https://doi.org/10.2766/20810.

\section{Notas biográficas}

Manuel Ángel Río Ruiz es profesor titular de Sociología en la Universidad de Sevilla. Especializado en sociología de la educación y de las relaciones interétnicas. Sus publicaciones académicas y divulgativas en estas áreas se encuentran y actualizan en la web entramados sociales.org.

Rubén Martín Gimeno es profesor asociado de la Universidad Pablo Olavide de Sevilla. Su labor docente e investigadora en el ámbito académico la alterna con su labor profesional donde ha desarrollado una trayectoria de más 15 años en la investigación social. Sus principales trabajos como investigador los ha desarrollado en el Instituto de Estadística y Cartografía de Andalucía en áreas como el estudio de las redes familiares, las trayectorias educativas desde una perspectiva longitudinal o la reflexión metodológica en la búsqueda de calidad de los datos en las encuestas. En la actualidad trabaja desarrollando su labor como Investigador Social en la Fundación Centro de Estudios Andaluces, desarrollando investigaciones para profundizar en distintos ámbitos de la realidad social de Andalucía y para mejorar y evaluar el impacto de las políticas públicas de la Administración andaluza.

María Ortega Gómez doctoranda en sociología por la Universidad de Sevilla. Miembro del grupo de investigación «Desigualdad social y Dominación en Andalucía». Fue Becaria de Colaboración y es Asistente Honoraria del departamento de sociología de la Universidad de Sevilla. Realiza su tesis sobre clases sociales y representaciones sociales de la Educación Infantil. 